\title{
Grand Challenges in Reconstructive Dentistry
}

\author{
Mutlu Özcan* \\ Division of Dental Biomaterials, Clinic for Reconstructive Dentistry, Center for Dental and Oral Medicine, University of Zürich, \\ Zurich, Switzerland
}

Keywords: reconstructive dentistry, dental biomaterials, clinical trials, digital dentistry, prosthodontics

Advances in the fields of biomaterials, biomedical engineering, and technologies continue to have a significant impact on patient care in almost all disciplines of clinical dentistry. Particularly during the last few decades, innovations in biomaterials, including reconstruction materials, oral implants, or regenerative materials, along with the implementation of digital technologies, changed the conventional facette of prosthodontics and narrowed the interface at least between Prosthodontics and Restorative Dentistry, which is the reason why, in some countries, the restorative discipline is referred to as Reconstructive Dentistry. Yet, regardless of the name of the discipline, the majority of the rehabilitations remain complex. For the most part, comprehensive cases are treated in this field in part due to the limitations in public healthcare systems or healthcare politics, late recognition of the problems, delayed dental visits due to economic reasons, or dental anxiety. That is why the dental profession still needs to restore the missing dental tissues in the oral cavity using dental biomaterials. Through many advances in dentistry and medicine, we have accomplished much, but we can achieve even more if we can overcome some challenges.

\section{NEEDS AND TRENDS}

The current dental needs of society are multi-dimensional. Fundamentally, the increased lifespan of human beings, constantly functioning aggressive oral environment, multimorbidity, changes in saliva flow and $\mathrm{pH}$, and the presence of biofilm and inflammation of the gums all result in loss of dental tissue. In addition to these factors, lifestyle changes, stress-related dental breakdowns, or caries also eventually lead to a need for restoration of the dentition. In recent years, the profession is also facing the emerging problem of dental erosion, a major cause for the loss of mineralized tooth structure even in the young population, as a result of extrinsic acid in erosive diet or intrinsic etiology, such as anorexia, bulimia nervosa, or gastric reflux.

Based on the current needs of the profession, from the technical perspective, dentistry as a whole is shifting towards faster, simpler, but more reliable, biomimetic, and minimally invasive yet affordable applications. Current trends are also in the direction of mass production, partially or fully computer-guided systems for tooth-, implant-, or mucosa-borne applications and individualized solutions. Although such trends may sound cutting edge, the primary requisite is in fact the integration of medicine and dentistry and implement the advances in a team approach. While doing so, early or timely detection, accurate diagnosis and treatment of systemic diseases, genetic disorders, drug-associated effects, aging, psychological and behavioral aspects, and their consequences on teeth, oral tissues, or craniofacial structures should be the principal goals and implementation of research.

Accepted: 04 March 2021

Published: 04 May 2021

Citation:

\section{CHALLENGES IN BIOMATERIALS SCIENCE}

Knowledge of dental biomaterials and dental technologies is not only needed for manufacturing reconstructions but also for rudiments of operation instruments, machines, computers, 
robots, etc. This covers firstly the fundamental physical, chemical, or biological aspects of the materials and then their clinical or technical applications. Dental biomaterials are usually developed in Research and Development departments of the industry, but their performance is further investigated at unbiased university settings primarily through fundamental biological, physicochemical, and mechanical functioning followed by testing on clinical or technical performance. Expertise in materials related to clinical dentistry has an effective role in decision-making when it comes to "conscious material choice" depending on the specific clinical situation. Personalized treatment options through correct material choice for the very patient undeniably requires this background along with biological and medical comprehension. Clinical trials where a material science background is not implemented and immature versions of materials are applied inevitably result in mechanical and biological complications requiring reintervention and repeated costly reconstructions. In the last decades, much effort has been spent on improving metallic, ceramic, and polymeric materials in Reconstructive Dentistry, and these are all still clinically indicated but present limitations in long-term intraoral function. In this context, the major problems include aging, wear, discoloration, fractures, surface degradation or biocorrosion of ceramics, polymers, and dental alloys, presenting challenges that we still need to tackle. Probably one of the most devastating current clinical challenges is facing the increasing incidence of periimplantitis and its consequences when it comes to the overall systemic health where pathology and genetic elements together with biocorrosion play a significant role in the etiology. No global solution has been established to date.

One of the biggest breakthroughs in dentistry is the developments in adhesion promoters and their implementation for minimally invasive applications. The use of adhesive promoters decreased the indications for invasive applications where tooth extraction could be avoided to a great extent and less or no tooth preparations are required through which restoration cycle could be reduced. While minimally invasive applications still need long-term clinical followup reports, reinforcing or regenerating the dentin to acidic attacks of microorganisms and inhibition of the degradation process at the tooth-adhesive interfaces are current challenges. Likewise, mimicking the anisotropy of natural teeth and bone structure using biocompatible regenerative materials and their long-term clinical stability certainly requires further investigation.

\section{CHALLENGES IN BIOMECHANICS AND BIOLOGY}

The long-term durability of tooth- and/or implant-borne reconstructions and removable applications is essential to the avoidance mechanical failures and thereby costly retreatments. In order to examine the durability aspect of materials, clinical trials are considered as the ultimate tests but they cannot pinpoint the true reason for failure due to the simultaneous impact of factors on restorations or implants in the oral cavity. The industry is also very fast in development, and, prior to clinical trials, standard battery tests are thus usually performed on given geometries followed by further testing the best outcome for complex geometries representing the structure of the dental applications in translational research settings such as the simulated oral environment. Unfortunately, often due to the marketing rush, thorough testing of reconstruction and implant materials do not often take place, frequently resulting in early clinical failures. Obviously, the mechanical and biological stability of reconstruction materials will remain one of the major focuses of research in the near future. Especially considering the aging population, expectations would increase for the long-term survival of dental rehabilitation, which will require optimization of dimensions and intrinsic properties of biomaterials from minimal to extensive reconstruction types processed either using conventional or digital technologies. In fact, the majority of the present failures could be circumvented in reconstructive dentistry when both fundamental material knowledge and processing techniques are mastered, and clinical trials are based on high-quality study design, from setting hypothesis to methodological aspects, which is unfortunately not always the case in most of the clinical trials.

\section{CHALLENGES IN DIGITAL TECHNOLOGIES}

On the production side in Reconstructive Dentistry, particularly for mass production, computer-aided design and computer-aided manufacturing (CAD/CAM) systems will surely continue to be used and improved but rapid prototyping processes based on additive technologies may overrule milling techniques in the near future. This processing path will only be effectively implemented in Reconstructive Dentistry with improved metallic, ceramic, and polymeric materials. One of the most important aspects of reconstructive rehabilitation is to generate an ideal treatment plan created on the basis of detailed data collection and subsequent analysis thus reaching the formulation of a complete diagnosis. With a univocal diagnosis, a treatment plan can be devised to solve the various problems of the patient in an optimal way, and it should take into consideration the expectations of the patient. Following data processing and the consequent formulation of the diagnosis, it is essential for the clinician to identify an optimal profile both from function and aesthetic perspectives. The digital workflows are enabling clinicians to combine all possible elements and the data collected during the diagnostic phase in order to obtain the "digital/virtual patient." Digital workflows and each step in the process require extensive research prior to full integration in the clinical trials. The field is also led by manufacturers, and currently immature systems are presently available while software programs undergo constant improvements. The digital workflow requires costly investments, and fundamental knowledge on the working mechanism of the systems should thus be considered in this dynamic field. In that respect, one big challenge is the extremely quick pace of implementation of newly introduced digital tools since the lifetime of such digital devices and innovation are getting much shorter. 
Against all advances in reconstructive dentistry, one other professional challenge and dilemma is the clash between the interface of delivering solutions to patients who "need" reconstructions versus fulfilling the "wish" of the patients who are largely under the influence of other media.

\section{CONCLUDING REMARKS}

Clinical decision making is dictated by multiple factors in dentistry. Frontiers in Dentistry: Reconstructive Dentistry welcomes high-quality research across the field of restorative and prosthetic dentistry with a focus on translational and clinical studies related to the reconstruction of intraoral defects, missing teeth, or dental tissues. This will include the application of dental biomaterials and technologies for the fabrication of tooth, implant-, or mucosa-borne dental appliances from a clinical perspective. This section will further provide information on the dynamic changes, offer solutions to challenges taking place in this arena, synopsize dental treatment options as well as protocols for patient care, and perhaps help revisit some of the doctrines in the profession in close collaboration with medical, biomedical, and basic science disciplines.

\section{AUTHOR CONTRIBUTIONS}

The author confirms being the sole contributor of this work and has approved it for publication.

Conflict of Interest: The author declares that the research was conducted in the absence of any commercial or financial relationships that could be construed as a potential conflict of interest.

Copyright (C) 2021 Özcan. This is an open-access article distributed under the terms of the Creative Commons Attribution License (CC BY). The use, distribution or reproduction in other forums is permitted, provided the original author(s) and the copyright owner(s) are credited and that the original publication in this journal is cited, in accordance with accepted academic practice. No use, distribution or reproduction is permitted which does not comply with these terms. 